\title{
Compromiso docente, una interpelación al sentido de la profesionalidad en tiempos de cambio
}

\author{
Teachers' commitment, an inquiry to the sense of professionalism in changing times \\ Compromisso de professores, uma interpelação ao sentido da profissionalidade em \\ tempos de transformação
}

Rodrigo Fuentealba Jara, ${ }^{a}$ Patricia Imbarack Dagach ${ }^{b}$

aUniversidad San Sebastián. Correo electrónico: rodrigo.fuentealba@uss.c1

bPontificia Universidad Católica de Chile. Correo electrónico: paimbara@uc.cl

\section{RESUMEN}

El actual contexto educativo sitúa al profesor como responsable a nivel de ejecutor del conjunto de disposiciones tendientes a favorecer los procesos de cambio en educación. Sin embargo, a partir de un enfoque que hace visible el compromiso docente y sus dimensiones es posible pensar desde una perspectiva en la que este actor, el profesor, pasa de ser un ejecutor de patrones establecidos externamente, a un actor protagónico, donde el compromiso se configura como un potente articulador de los procesos identitarios y promotor de profesionalidad que otorga sentido, propósito, implicación y acción a los procesos de cambio.

Palabras Claves: sentido de propósito, identidad profesional, profesionalidad, desarrollo profesional.

\begin{abstract}
The current educational context sets the teacher in a level where he/she is the responsible and executor of actions tending to favor the processes of change in education. However, from an approach that makes visible the teacher's commitment and its dimensions it may be possible to think from a perspective in which this actor, the teacher, happens to be an executor of externally established patterns -a leading actor- where commitment is configured as a powerful articulator of identity processes, as well as, promoter of a professionalism that gives meaning, purpose, implication and action to processes of change.
\end{abstract}

Key words: purpose sense, professional identity, professionalism, professional development.

\section{RESUMO}

O atual contexto situa o professor como o responsável pela execução do conjunto de disposições destinadas a facilitar os processos de transformação na educação. No entanto, a partir de uma abordagem que evidencia o compromisso dos professores e suas dimensões é possível pensar a partir de uma perspectiva na qual esse ator, o professor, passa de executor de padrões estabelecidos externamente a ator principal, onde o compromisso se configura como potente articulador dos processos de identidade, bem como promotor de profissionalidade que dá sentido, propósito, implicação e ação aos processos de transformação.

Palavras-chave: sentido de propósito, identidade profissional, profissionalidade desenvolvimento profissional. 


\section{INTRODUCCIÓN}

Ser docente se afecta, retroalimenta y re-significa a partir de los cambios en el contexto y las influencias sociales. Cambios que actualmente exigen a los profesores enfrentar a diario nuevas exigencias, que nacen de una clara orientación a los resultados y que ponen en un difícil panorama el quehacer del profesor (Consejo Económico y Social, 2011). Las posteriores reflexiones darán cuenta del "compromiso docente", dimensión del desarrollo profesional recientemente investigada, que actúa como un potente sostén de la identidad y profesionalidad en tiempos de cambio, develando la forma en que los docentes y las escuelas gestionan su accionar y espacios de desarrollo dentro de una realidad social dinámica. Se asume que, desde la lógica del compromiso, es posible construir espacios propicios de implicación e incidencia en el diseño de política con la profesión, la enseñanza, los estudiantes y la institución escolar.

Bajo lo planteado, el texto se estructura en cuatro apartados que sitúan la necesidad de considerar aspectos que permitan concebir al profesor como parte indispensable en los procesos de cambio, no pensándolo como destinatario y ejecutor, sino que como parte fundamental del conjunto de acciones que determinarán nuevos rumbos en el quehacer educativo.

Así, en un primer apartado se hace referencia a las principales características del actual contexto educativo y de la necesidad de pensar vías alternativas para situar el problema educativo. En un segundo apartado, se hace referencia al compromiso docente como posible vía para pensar los procesos de cambio y su incidencia en la identidad docente, procesos que de manera articulada dan cuenta de la emergencia de sentido de propósito y se constituyen en catalizadores de los procesos de cambio; para luego, en un tercer apartado, asumiendo la necesidad de considerar la dimensión subjetiva que reconoce al compromiso docente como elemento central ante la necesidad de cambio, preguntarse por la manera en que dicha consideración pudiese tener cabida en las futuras construcciones de política educativa que conciban, desde una mirada amplia, lo que significa ser profesor, su profesionalidad y su desarrollo profesional. Finalmente, en un cuarto apartado, se vislumbran caminos que nos permitan plasmar efectivamente dichas posibilidades.

\section{NUEVAS DEMANDAS EN EDUCACIÓN: TIEMPOS DE CAMBIO}

A partir de la década de los 90 con el advenimiento de la democracia, la mayoría de los países latinoamericanos ha sido testigo de grandes transformaciones en las formas de comprender y re-pensar el sistema escolar, sus fines y metas. Las claras prerrogativas de instituciones internacionales ${ }^{1}$ han movilizado cambios y reformas en la mayoría de nuestros países. Orientaciones que en ocasiones desconocen las particularidades del sistema educativo nacional y que, por ende, tensionan la noción de profesionalismo y profesionalidad, situación que afecta directamente tanto a la institución escolar que "deja de ser una institución al servicio de proyectos culturales compartidos, para constituirse en una organización orientada a producir y enseñar competencias útiles para los individuos y a generar títulos que certifiquen

Banco Mundial. Programa de las Naciones Unidas para el Desarrollo (PNUD), Organización de las Naciones Unidas para la Educación, la Ciencia y la Cultura (UNESCO, 2011) entre otros. 
dichas competencias" (Martinic, 2010: 64), así como al docente que en ella se desempeña, quien pasa de ser un actor protagónico a un actor pasivo y sujeto a cambios externos.

Esta tensión está marcando la experiencia actual de los profesores, interpelados por los nuevos mapas que prometen las políticas de incentivo, desempeño, estándares, entre otros $^{2}$. El presente énfasis exhorta a un creciente efectivismo que pone acento en el "hacer" y "producir" y que ofrece moldes identitarios que se alejan de las formas en que el docente se comprende, define y actúa (Sisto, 2011: 179). En la emergencia de este paradigma efectista, la institución escolar es exigida a dar respuesta a expectativas y demandas por resultados 3 . De igual forma, el docente está demandado a responder de manera "eficiente", no necesariamente considerando lo que ello significa en sus contextos de desempeño, ni tampoco lo que implica en la figura del profesor.

La existencia de una política verticalista, top-down, tensiona los procesos de individuación de la escuela y pone en entredicho el sentido de ésta, afectando directamente los modos de actuar de los profesores. En este contexto "se interpela directamente a los profesores, su principal fuerza de trabajo, como responsables ya no de prácticas pedagógicas y formativas concretas, sino del desarrollo económico y social de los países” (Sisto, 2011: 179).

Dicha tensión, expone al profesorado a procesos de negociación constantes entre la adopción irreflexiva de la política de turno, que demanda el "hacer" del profesor, y la práctica pedagógica que valoriza el "ser" del docente en tanto sujeto de juicio, comprometido y socialmente situado. De esta forma, en la experiencia de dar cuenta sobre resultados, los actores sociales, y particularmente los docentes expuestos a políticas de apoyo externo, pueden desarrollar, como mecanismos de estructuración, juegos de intercambio de aceptación recíproca o sumisión estratégica que generalmente conllevan altos costos para ambas partes y que, incluso, pueden impulsar a que los mismos actores sociales construyan prácticas que hagan fracasar la política (PNUD, 2009). ${ }^{4}$

En este sentido, la articulación de políticas que conduzcan a mejoras en la calidad no puede depender en forma exclusiva de la experticia de operadores políticos, de la ejemplaridad del diseño, de recursos ni de la magnitud de la propuesta. Acortar la brecha entre la formulación política y su materialización en espacios concretos, más aún, en contextos de cambio, dependerá en buena medida de las estrategias y alianzas que definan en conjunto modos de actuar por parte de los actores sociales, que emanan directamente de una mirada que asuma no sólo la profesionalidad docente, sino que también el conjunto de componentes que configuran la esencia del ser profesor. Es así como el éxito o el fracaso

En nuestro país, esto se materializa en un conjunto de esfuerzos por gestar mejoras en el sistema escolar, a través de programas de apoyo (ej. Programa de Apoyo Compartido, supervisión, mejoras en la infraestructura, materiales entre otros); en unión a sistemas de estandarización, evaluación y "responsabilización" (accountability), especialmente desde la entrada de la Subvención Escolar Preferencial y la Agencia de la Calidad, elementos que consolidan un modelo de apoyo más presión.

3 Vale la salvedad que tanto enseñanza como aprendizaje responden a una doble realidad. Por una parte, hacen mención al carácter privado, exclusivo de la interacción profesor-alumno, y por otra, a un espacio público en el que la práctica pedagógica es objeto de valoración de resultados y logros académicos que son compartidos por la comunidad educativa. Esta investigación se circunscribe a la primera aproximación, la que en palabras de Day (2006) asume la enseñanza y el aprendizaje como una actividad netamente humana, que tiene que ver con valores, identidades y fines morales que orientan el actuar del docente, pero al mismo tiempo las actitudes ante el aprendizaje de profesores y estudiantes, sus preocupaciones y compromiso.

$4 \quad$ Ejemplo de ello es la propuesta de reforma del actual gobierno, que si bien no direcciona ni específica la orientación del cambio, afirma que "Es necesario definir qué entenderemos por calidad y cómo la mediremos. Esto requiere que la profesión docente esté dentro de las más valoradas socialmente y de las más exigentes, así como también entregar el espacio y las herramientas necesarias para que los profesores puedan desarrollar su labor". 
dependerá en buena medida del reconocimiento del docente como un actor más dentro de las transacciones simbólicas entre los actores, bajo el supuesto de que el diseño de una política orientada a la mejora del sistema educativo en su conjunto debe dirigir la atención no sólo a la manera concreta en que se hacen las cosas, a las acciones y relaciones en situaciones específicas ${ }^{5}$, sino que también al modo de implicación y definición por parte de los profesores, particularmente en tiempos de cambio.

Como alternativa se propone re-pensar la política educativa visibilizando la subjetividad de los actores, espacio en el que profesionalidad e identidad profesional se nutren orgánicamente para marcar diferencias en sus contextos de desempeño. De ahí la relevancia de pensar las reformas educativas a partir de lo que significa que la responsabilidad de levantar el estándar de calidad, diseñar políticas e implementarlas no puede eludir lo que implica ser docente ni la participación de estos en el diseño de procesos de reforma. Al poner acento en los modos de actuar y relacionarse propios de la práctica educativa no sólo se recogen dimensiones subjetivas del quehacer del profesor, sino que se explicitan los distintos espacios de actuación de los docentes. Dichos espacios tienen que ver, entre otras cosas, con disposiciones que se generan en la relación que establece el docente con sus estudiantes, en la fuerza de identificación con la enseñanza, con la institución en la cual se desempeña y con las oportunidades de desarrollo profesional a las que accede.

Estos ámbitos pueden ser leídos en tres dimensiones (institucional, personal y profesional) que engloban lo que recientes aproximaciones denominan "Compromiso Docente", y que emerge en la discusión académica como un poderoso articulador de la identidad profesional, de la esencia de la docencia (Darling-Hammond, 2009; Hargreaves et al., 1998; Van de Berg, 2002; Vezub, 2007), señalando que "los futuros docentes deben tener no sólo buenos resultados y excelentes habilidades interpersonales, sino que también un profundo compromiso personal para enseñar y trabajar en las escuelas" (Darling-Hammond y Rothman, 2011: 13). También Razak, Darmawan y Keeves (2009) coincidieron en que el compromiso de los profesores es de vital importancia en una educación de calidad. En este sentido, el compromiso docente ha sido identificado como uno de los factores más críticos para el éxito futuro de la educación y las escuelas (Selamat y Nordin, 2014). Asumir el compromiso desde dicha perspectiva es central para "comprender cómo los profesores definen y re-definen su identidad profesional, cómo encaran tanto las reformas como las demandas cotidianas en diversos contextos y situaciones sociales complejas" (Ávalos et al., 2010: 237).

La lógica del compromiso hasta aquí expuesta otorga sentido a una concepción de lo que se entenderá por profesionalidad, entendida en un sentido amplio que incluye aspectos propios de la enseñanza como profesión, es decir un proyecto pedagógico que se vincula con aspectos éticos y epistemológicos y que afectan no sólo la práctica, sino que también la identidad personal y colectiva del docente (Pérez-Gómez cit. en Pérez-Gómez, Barquín y Angulo, 1999). Desde esta perspectiva, nuestra reflexión nos dirige hacia una mirada del compromiso que, junto con establecer vínculo con la identidad del profesor, se asocia al mismo tiempo con el quehacer del docente, lo que vendría a ser una forma renovada de conceptualizar el Desarrollo Profesional Docente.

La centralidad del rol del profesor pasa, entre otras dimensiones, por la idoneidad de su práctica pedagógica, ya que ésta puede explicar la mayor parte de la variación observada en los resultados de sus estudiantes (Kuijpers, Houtveen y Wubbels, 2010) y "existe creciente evidencia de que, luego de las condiciones socioeconómicas y culturales de las familias de los alumnos y de la composición de estas características entre sus compañeros, es el docente quien determina las oportunidades de los estudiantes para adquirir conocimientos y habilidades" (Bellei y Valenzuela, 2011: 257). 


\section{COMPROMISO Y PROFESIONALIDAD}

Precisar el constructo compromiso y su relación con la profesionalidad es gravitante para lograr una mirada comprensiva del desarrollo profesional en un escenario de cambio educativo. "Compromiso" es un término utilizado a menudo por los profesores tanto para describirse a sí mismos o entre pares, como para hacer referencia a su identidad profesional. Comúnmente, este concepto es empleado por ellos para caracterizar los atributos deseables en un profesor (Crosswell y Elliott, 2004). Sin embargo, a pesar de lo cotidiano de su uso, es un constructo polisémico, usado indistintamente para hacer referencia a la forma en que los profesores se desempeñan, es decir, profesores que realizan un "buen trabajo docente" (Abd y Ngurah, 2010); al tipo de implicación con una tarea, como sinónimo de profesores "dedicados" (Tang y Choi, 2011); como sentido de pertenencia en referencia a un determinado ethos profesional ${ }^{6}$; o como forma de comportarse que define la profesionalidad docente en función de una pasión por la enseñanza"7.

El compromiso es generalmente considerado como un atributo deseable, asociado a un sentido de profesionalismo ${ }^{8}$ que Choi y Tang (2009) definen como vínculo psicológico que tiene implicancias tanto en la actitud como en el comportamiento de las personas y que les permite voluntariamente realizar considerables esfuerzos en pos de beneficiar aquello de que es objeto de compromiso, lo que en el caso de la docencia se traduce en profesores que están dispuestos voluntariamente a entregar recursos a favor del ejercicio de la docencia. Gupta y Kulshreshtha (2009) complementan lo ya mencionado enfatizando que el compromiso alude, entre otras cosas, a dedicación a la tarea, adhesión a los objetivos de la educación, una profunda preocupación por sus estudiantes, conciencia de la responsabilidad y del rol docente y, finalmente, un alto grado de profesionalismo.

A fin de precisar el término, Nias (1981) concluye que el compromiso es la cualidad que distingue a quienes se perciben como "auténticos docentes" de quienes tienen sus principales intereses en ocupaciones fuera de la escuela. Así, desde esta perspectiva, permite diferenciar a aquellos profesores que "se entregan" de "quienes no evidencian una preocupación por sus estudiantes". Esta dimensión es igualmente validada por los propios alumnos, quienes identifican a la base de una buena enseñanza a aquellos docentes que se preocupan por ellos y que comparten su ejercicio profesional con una autentica inquietud por quienes les son confiados, en tanto bienestar general y orientación al logro académico (Day, 2009).

Las particularidades del compromiso se evidencian en "profesores que creen firmemente que pueden marcar una diferencia en el aprendizaje y resultados de sus estudiantes por lo que ellos son, lo que ellos saben y cómo ellos enseñan" (Day, 2007: 254); asimismo los profesores comprometidos tienen la certeza de que tanto ellos -su identidadcomo su trabajo -conocimiento pedagógico, disciplinar, profesional-; las estrategias de enseñanza que utilizan, así como también sus creencias, actitudes y valores, tendrán un impacto significativo en sus alumnos e instituciones en las cuales trabajan (Day, 2007,

El ethos no solo son formas de comportarse, sino también convicciones o creencias de orden axiológico, deontológico y ético.

La pasión se asocia con un fuerte afecto positivo, satisfacción, experiencia de flujo, vitalidad y control de propósito en la elección para iniciar y detener la actividad (Moè, Pazzaglia y Ronconi, 2010).

8 Profesionalismo, que si bien no niega la condición de conocimiento práctico de la docencia similar al de las artes, sustenta el estatus de la docencia como profesión en el logro de tres factores centrales: una base de conocimientos específica, autonomía en el ejercicio de la profesión frente al sistema social y control sobre la calidad del ejercicio de la docencia (Ávalos, 2002). 
2009; Sammons et al., 2007). Así descrito, el compromiso es una dimensión descriptible que puede ser intencionada en los procesos de formación docente y que se asume como aprendido en interacción con otros y en un determinado contexto, lo que hace directa referencia a su carácter situado (Abd y Ngurah, 2010; Crosswell y Elliott, 2004); por lo tanto, no puede ser considerado como lujo, adorno, posesión o competencia de solo unos pocos profesores, ya que es esencial para una buena enseñanza y, en consecuencia, por incómodo que pueda parecer, está en el centro de lo que ella es (Day, 2006).

Si asumimos el compromiso docente como el nivel de adhesión e identificación del profesor a determinados aspectos de su quehacer, es posible afirmar que hay diversos ámbitos que pueden ser objeto de compromiso para un profesor y que incluso, a partir de prioridades o demandas, pueden cambiar a lo largo de la trayectoria docente y en los diferentes contextos de desempeño. Así, para Klassen y Ming (2010) el compromiso docente implica muchas facetas que incluyen diversos objetivos relacionados con el trabajo, entre ellos: con la organización, la ocupación, los pares, entre otros. Atak (2011) identifica tres dimensiones del compromiso docente, dependiendo del objeto de interés del profesor y los espacios en los cuales está dispuesto a realizar una mayor inversión de tiempo y energía: compromiso con la organización, compromiso con la profesión de la enseñanza y compromiso con el aprendizaje de los estudiantes, independientemente de sus dificultades académicas u origen social. Siguiendo a Kwan (2012) el compromiso de los docentes generalmente se expresa en tres dimensiones: compromiso con la escuela, compromiso con la enseñanza y compromiso con los estudiantes.

Teniendo en consideración lo hasta aquí mencionado, podemos afirmar que para abordar comprensivamente el compromiso docente se debe atender a la compleja articulación de relaciones que el profesor establece con la enseñanza, sus estudiantes, profesión y escuela, lo que distingue dimensiones en las cuales se expresa dicho compromiso y que ponen en el centro de éste un fuerte componente relacional. De esta forma su construcción y consolidación dependen de las relaciones con, para y por otros, las que se solidifican en y a través de un contexto y sentido de propósito particular.

Figura 1. Compromiso Docente: sistematización de los elementos teóricos

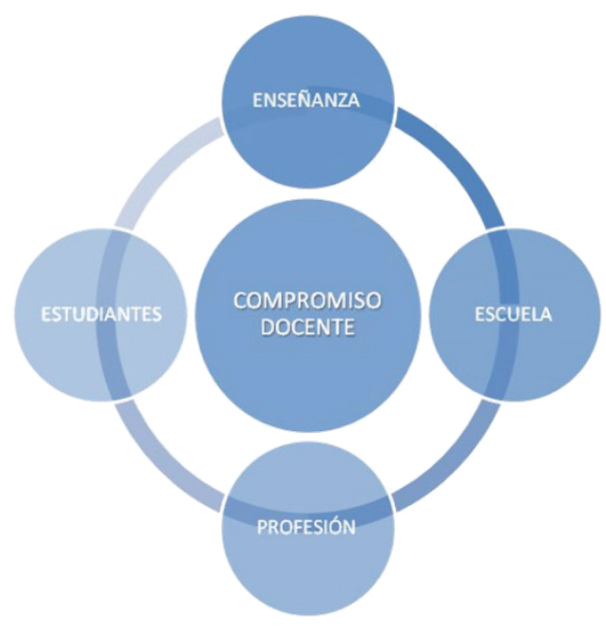


El compromiso reviste, define y configura el ser del profesor más allá del rol de transmisor de aquello producido por otros, pues antes precisa la esencia de ser profesor. De esta forma el compromiso incide como eje que responde a lo que el docente es, tanto en lo individual como en lo colectivo, es decir aparece como un catalizador de la identidad profesional del profesor.

Lo gravitante del compromiso no sólo tiene que ver con el constructo en sí mismo, sino en la importancia que reviste en la conformación de la profesionalidad docente. El compromiso provee de elementos al profesor en la articulación de una descripción coherente respecto de sí mismo, en sintonía con las estructuras sociales en las que se desempeña y las formas de actuar que adopta (Day y Qing, 2010), toda vez que el "el saber docente es de naturaleza social porque se adquiere en el contexto de la socialización profesional, es decir en las relaciones que los profesionales establecen en los contextos en los cuales participan" (De Azevedo, 2013: 100), a lo cual es necesario agregar que la naturaleza social de la docencia le permite afectar/transformar y, al mismo tiempo, ser afectado/transformado por lo social, por los otros. Ello pone especial acento en la construcción de identidad profesional ${ }^{9} \operatorname{como}$ componente esencial de la profesionalidad docente, la cual hemos asumido determinada y configurada por el compromiso como rasgo particular en la definición de todo docente.

La manera en que los profesores forman su identidad profesional está influida, depende y se basa en la existencia e interacción con "otros" 10 , espacio propicio para la construcción identitaria, toda vez que dicha interacción exige acentuar semejanzas y diferencias en función de referentes externos (Larraín, 2005) sobre los cuales puede discernir cómo se siente acerca de sí mismo, o ponderar su autovaloración y conocimientos propios, pero, al mismo tiempo, indagar respecto de cómo lo perciben sus estudiantes, pares y directivos. En síntesis, son los otros significativos y/o próximos quienes en el día a día insuman al docente de argumentos y relatos que le permiten construir una narrativa respecto de quiénes son, este último aspecto base para realizar ajustes pertinentes y efectivos a su práctica (Day, 2009; Ávalos y Sotomayor, 2012).

La interacción simbólica con otras personas, que refiere Larraín (2005), remite a una doble entrada ante la narrativa identitaria. Por una parte, la identificación como fuerza unitiva que se fundamenta en el rol de los modelos de referencia con los que cuenta el docente, y sobre los cuales se busca semejanza, proceso, por lo demás, saturado de componentes vinculados al compromiso como eje de identificación con la profesión docente. Desde los procesos de identificación, el compromiso actúa como un poderoso imán que favorece la vinculación con la docencia en la construcción de la identidad profesional. Por otra parte, los procesos de identización aglutinan elementos de diferenciación biográfica o personal que ponen en evidencia el quehacer docente en el centro identitario, que definen y configuran quién es y cómo es ser profesor (Galaz, 2011).

En el juego entre identificación e identización se entrelazan identidades previas y nichos culturales y sociales, ambos determinan posibles modos de ser, lo que Monereo y Badia (2011) denominan "selfway", y que dotan a la profesión de un discurso que

Comprendida como "un proceso de construcción en la que los individuos se van definiendo a sí mismos en estrecha interacción simbólica con otras personas” (Larraín, 2005: 90-91).

10 Siguiendo a Monereo y Badia (2011) la identidad, definida en un sentido estricto, comienza cuando el ser humano es capaz de auto-reconocerse y de atribuir determinados rasgos, conductas y consecuencias de las acciones a sí mismo. Incluso en los niños, antes de la emergencia del yo, está el reconocimiento del otro. 
legitima y valida el quehacer docente en base a competencias, valores y desafíos que "poseen un carácter particular y privativo y que permiten el dominio (percepción de sí) y el reconocimiento (social/oficial)"11 (Galaz, 2011: 91).

Sobre el selfway o reconocimiento social/oficial que discute Galaz, el compromiso docente hace referencia a un determinado ethos profesional, o forma de comportarse, que define la profesionalidad docente en función de una "pasión por la enseñanza", entendida como un fuerte afecto positivo, satisfacción, experiencia de flujo, vitalidad y control en la elección para iniciar y detener la actividad (Moè, Pazzaglia y Ronconi, 2010).

Ante las múltiples narrativas y/o representaciones que puede haber construido el docente en su interacción con otros, la pregunta por el "ser del profesor" es articulada y convocada por el compromiso como eje central que ofrece al docente la posibilidad de ser uno y muchos al mismo tiempo, y de proteger una "identidad" que dota de permanencia y estabilidad a su "modo de ser profesor" (Monereo y Badia, 2011).

\section{HACIA UNA PROFESIONALIDAD COMPROMETIDA, HABLEMOS DE POLÍTICA}

Se ha fundamentado a favor del compromiso docente como eje identitario: "la fuerza pedagógica de los profesores depende en gran parte de la fascinación y encantamiento por lo que hacen" (López de Maturana, 2010: 59), ${ }^{12}$ razón por la cual el compromiso aglutina atributos deseables que de suyo se asocian a un fortalecimiento del carácter de la profesión a través de un "modo de ser compartido", sin descuidar la complejidad, sofisticación y multidimensionalidad del constructo; entonces, ¿es posible pensar el diseño de políticas de desarrollo profesional considerando el compromiso?. Resolver esta pregunta resulta central, ya que las políticas intentan -más aún en materia educativa- responder al diseño de una acción compartida e intencionada, fruto de decisiones e interacciones que definen el curso de acción a un conjunto de actores al tratar un problema de interés (Lahera, 2004).

Hemos ejemplificado la actual demanda de políticas educativas que promueven la construcción de lo que Bolívar denomina un "estado evaluador", globalizado y que persigue comparar resultados inter-países, "siendo atrapado o colonizado por la lógica mercantil, común para los gobiernos conservadores y los de la izquierda neoliberal" (Bolívar, 2008: 57). El escenario descrito puede dar paso a un compromiso que implica una vinculación al ejercicio docente en función de requerimientos externos que determinan una práctica en base al logro de resultados (Lisbona et al., 2012; Santoro, Pietsch y Borg, 2012), lo que desborda la identidad y deriva en una sobre identificación con la docencia, permitiendo que la acción pedagógica sea valorada por encima de otros intereses, desestimando la participación y llegando a experimentar conflicto entre la docencia y otras actividades e intereses (Chamarro et al., 2011: 343).

11 La distinción entre identidad, autoconcepto y self es difusa y de común se utilizan de forma indiferenciada, sin embargo, Álvarez y Badia (2011) identifican la identidad con aquellas características más permanentes que el profesor se auto-atribuye y sus acciones en escenarios particulares de acción educativa, que correspondería al self. En paralelo, el autoconcepto puede ser comprendido con la forma en que el docente refiere y/o narra respecto de sí mismo en un relato articulado y coherente que construye identidad.

12 Investigaciones recienten confirman en una muestra de 251 docentes que no cambiar la decisión por la carrera y la satisfacción con la profesión son factores gravitantes en la comprensión del compromiso docente (Imbarack, 2013). 
La exclusiva presencia de determinantes externos en el compromiso levanta una mirada de sospecha respecto de la forma en que se estructura, construye y propone actualmente la política educativa, de suyo en torno a una reforma en base a estándares, paradigma que impulsa sistemas centralizados de aseguramiento de la calidad educativa y que la definen desde el logro de aprendizaje de los estudiantes. Dicha mirada convoca a un sistema que se estructura en función de disciplinas valoradas como centrales y medibles, privilegiando la responsabilidad ante resultados y la rendición de cuentas (Espínola y Claro, 2010). Estos elementos conjugan un escenario en donde la pregunta por cómo el docente resignifica dichas prerrogativas y requerimientos externos es esencial en la definición del tipo de compromiso a asumir.

Sin desconocer la necesidad de regular el sistema educativo y definir políticas que orienten los requerimientos básicos, la salida ante esta tensión no implica retroceder y desconocer estándares de desempeño ni perfiles de egreso que regulen el trabajo docente, sino más bien y en mayor medida repensar cómo éstos se ajustan, acomodan y configuran en función de ser del profesor, lo que posibilitaría una apropiación igualmente comprometida y armónica de la política educativa, tanto en su diseño como en su implementación.

En este contexto adverso, la capacidad del docente de responder constructivamente a las tensiones propias de su quehacer, que actualmente le son inherentes, se transforma en una herramienta clave que fortalece y mantiene el compromiso, ya que implica que el docente desarrolla su trabajo porque elige hacerlo, y no en función de presiones y requerimientos externos; tampoco en base a resultados, aprobación social, reconocimiento, logro de aprendizaje o cualquier otro elemento que impulse externamente el trabajo que realiza, sino más bien porque el trabajo es importante y significativo para él. En tal medida, su labor deja de ser simplemente una actividad e influye en la configuración de la identidad y transformándose en parte importante del autoconcepto ${ }^{13}$. Lo expresado puede ser leído tanto a nivel individual como colectivo.

La relevancia del compromiso docente, que lee e interpreta desde su incidencia en la identidad y profesionalidad los requerimientos externos, es lo que gesta en el profesor la fuerza necesaria para implicarse libre y voluntariamente, ocupando el compromiso un lugar importante en la identidad, sin dañarla, lo que le permite incidir con flexibilidad y apertura en la política (Vallerand, 2012). Esta lectura sitúa la figura del docente como un profesional autónomo, con voluntad y disposición permanente de decisión, que se aleja tanto de una rígida esclavitud a las formas como de una arbitrariedad que desconoce las normas.

Ante lo descrito, parece ser clave la distinción respecto de qué marca la diferencia, qué elementos disciernen respecto del curso que toma el compromiso docente ante la política educativa a fin de evidenciar las complejidades de una práctica saturada de una vivencia subjetiva de malestar, la que se justifica, asume y sobrelleva desde un compromiso que mal entiende el vínculo docente con la práctica pedagógica en todos sus ámbitos de expresión: enseñanza, estudiantes, institución, profesión.

Puesto en discusión, el tránsito y la movilidad que permiten desplazar el compromiso docente desde un vínculo pernicioso hacia un compromiso armónico, se comprenden desde la tensión entre la conservación de la identidad y la necesidad del docente de trascender sus propios límites y volcarse hacia el exterior, a través de un fuerte, sólido y claro sentido

"La Pasión se puede liberar cuando las personas ven sus roles y responsabilidades en un sentido más amplio que un simple trabajo" (Funk, 2008: 8). 
de propósito (Jacobo, 2003). Dicho sentido de propósito refiere una forma particular de vincularse con cada una de las dimensiones que encierra el compromiso docente: enseñanza, estudiantes, profesión y escuela; y aun cuando no es el centro, sí es posible afirmar que se ubica en el eje de estas dimensiones entendido como impulso interior que da oportunidad al profesor de actuar según una decisión personal, transformando la práctica docente en acciones articuladas más allá de la obligación, pues antes bien lo hace en función de objetivos interpersonales que inducen al docente a determinadas acciones y dotan de sentido el actuar del profesor. Es, por tanto, la participación en la construcción de un sentido de propósito donde el docente se compromete, vincula e implica con la labor que realiza a diario.

Lo que el sentido de propósito hace es, por una parte, acentuar la mirada no sólo desde el ámbito personal, en el quehacer docente; y por otra, poner de relieve que "el profesor, como persona, es la esencia por la cual, la educación en sí misma tiene lugar" (Korthagen, 2010: 96), dimensión actualmente reconocida e investigada ampliamente en diversos contextos de formación docente (Bolívar, 2010; Cassasus, 2009; Elmore, 2010; Tigchelaar, Brouwer y Korthagen, 2008). Cada vez más, tanto los investigadores como los formadores de profesores lo reconocen: "la buena formación no puede reducirse a las técnicas; la buena formación viene de la identidad y la integridad del profesor" (Palmer, 2007: 10).

Reconocer el asidero del compromiso docente pone en discusión la importancia de acentuar la integración entre dos dimensiones: lo personal y lo profesional; lo que el docentes "es" y lo que "hace" al servicio de una práctica de calidad ${ }^{14}$. Dicha integración posibilita la adopción de una perspectiva distinta, que hemos denominado "desde dentro", a fin de comprender y analizar los sentidos y valores vinculados al trabajo educativo que asumen y desarrollan los propios docentes dentro de un contexto de desempeño complejo compuesto por múltiples actores (desde el Estado, representantes políticos nacionales, regionales y locales, la comunidad educativa y nacional, etc.) y una diversidad de intereses y demandas.

Bajo esta perspectiva, sería pertinente considerar el compromiso como parte de la construcción de política, discutiendo previamente si su diseño debería considerarlo como un imperativo, de manera de prestar atención a las acciones que articulan y aseguran su presencia o, más bien, considerar el compromiso docente como un desiderátum.

$\mathrm{Al}$ asumir el compromiso docente como un imperativo es deseable su emergencia en contextos de formación y desempeño docente, en tanto responde a un modo específico de "ver al profesor" y su relación con la educación. Bajo este supuesto, es necesario contar con estrategias y políticas que aseguren su presencia en quienes optan por la docencia y, ciertamente, en aquellos que la ejercen, entonces ¿es proyectable su inclusión en una política donde priman estándares de desempeño? Una precisión a este respecto: el concepto de estándar hace referencia a lo que el docente debe saber y poder hacer para "ser considerado competente en un determinado ámbito. Los estándares señalan un "qué", referido a un conjunto de aspectos o dimensiones que se debieran observar en el desempeño" (Ministerio de Educación

\footnotetext{
Hoy en día, experiencias en relación al hacer del profesor se aglutinan en torno al rediseño de la institucionalidad educativa, que a través de la superintendencia de educación y la agencia de calidad, fija estándares de calidad previamente definidos y exige responsabilidades en la rendición de cuentas a las escuelas y, en ellas, a los docentes. Ejemplo de ello es la actual Ley de Subvención Educacional Preferencial (SEP), el Programa de Apoyo Compartido (PAC), políticas y programas como el P-900, liceos prioritarios, entre otras políticas, cuyo fundamento es el principio de discriminación positiva, a propósito de la equidad, en la asignación de recursos y en el diseño de iniciativas que compensen a través de la inclusión de un marco regulatorio del hacer del docente, mejores prácticas y, por ende, el logro de mejores aprendizajes. En paralelo, se elevado la inclusión en el sistema educativo de estándares complementarios que sirven para modelar y promover entre los actores educativos (evaluación docente y prueba INICIA entre otros).
} 
de Chile, 2011: 5), y en paralelo abordan dimensiones del "cuánto" que permiten sopesar la distancia o cercanía en el quehacer docente de aquello que se evalúa como deseable ${ }^{15}$.

En independencia de la discusión respecto de los beneficios, o no, que reporta delimitar una política educativa en base a estándares, poco se sabe de los efectos que la inclusión per se de normas, patrones o modelos tiene en materia educativa. La evidencia sugiere que la creación de estándares por sí solos tiene poca probabilidad de influir en la práctica docente de manera significativa (Hamilton, Stecher y Yuan, 2008); más bien, lo que se requiere es conjugar un complejo y delicado equilibrio entre prescripción y desarrollo entre autonomía y heteronomía, entre regulación y apoyo que la sola política desconoce.

El recorrido habitual de una política orientada a estándares da paso a una reforma en base a pruebas estandarizadas, lo que agudiza las tensiones antes mencionadas ya que conduce y perfecciona un sistema en el que una prueba, en lugar de las normas, comunica expectativas y prácticas. En el actual sistema nacional de aseguramiento de la calidad -política y estructura recientemente estrenada en nuestro país- se pronostica la definición explícita de metas o estándares fijados a cada actor educativo, cuya existencia preconcibe la creación de sistemas formales de evaluación y, por lo tanto, la aplicación de sanciones y medidas correctivas vinculadas a resultados. En este escenario las sanciones se unen a productos específicos, medibles; de esta forma las prácticas tienden a distorsionarse, transformando la asociación entre compromiso y estándar en un camino inviable de recorrer, toda vez que el estándar -la prescripción- coarta el compromiso docente como forma de comportarse que define la profesionalidad en función de una pasión por la enseñanza (Choi y Tang, 2009).

¿Es posible a través de la definición de un estándar abrazar la promesa de aquello que ha de desarrollarse en el profesor? ¿Es factible incorporar el compromiso como estándarimperativo en la formación y ejercicio docente? Conviene distinguir que lo inapropiado de transformar el compromiso docente en un estándar que haga referencia a lo esperable pasa por reducir "la calidad" de la práctica pedagógica a un punto de corte, a un valor y a una dimensión, cuando en realidad la constituye, tal como hemos argumentado, antes se trata una inclinación a autodefinirse en función de una actividad que se quiere (o incluso ama), que se valora como importante y en la cual se invierte tiempo y energía en forma regular (Vallerand, 2012; Vallerand et al., 2010).

Desde otra perspectiva, haciendo una lectura del compromiso docente como un desiderátum, palabra latina que refiere a desidere "algo deseado"16, éste es también prerrequisito, necesidad, pues se alude a lo esencial o lo que todavía no se ha vivido, todas expresiones que contienen en sí la idea de desarrollo, entendida como potencia, es decir que posee en sí misma la predisposición a desarrollarse ${ }^{17}$. Desde esta perspectiva, la comprensión del compromiso docente se hace viable y perfectible desde la concepción aristotélica que distingue "acto" de "potencia", dos formas diferentes de comprender el ser. El compromiso en "acto" adopta características, propiedades o determinantes en función

\footnotetext{
15 El documento citado continúa y afirma que los estándares permiten develar la profundidad y complejidad de la enseñanza, destacando aspectos que resultan indispensables y decisivos para la efectividad del quehacer docente, lo que deriva en una oferta inconclusa de la política en base a estándares.

16 Recordemos que el compromiso es utilizado por la mayoría de los profesores para hacer referencia a atributos deseables en un docente (Crosswell y Elliott, 2006).

17 La descomposición del vocablo nos lleva a su mejor comprensión "de" (abajo) "sider o sidus" (estrella), es decir ir más allá en función de una meta, sin desconocer la relevancia del recorrido.
} 
del "presente". Ahora bien, el "acto" transita a la "potencia", hacia aquello que aún no es, pero que apunta a un ser en virtud de lo que ya es, del "acto". El compromiso en potencia contiene ya el compromiso en acto, y a su vez el compromiso en acto es promesa de aquello que ha de desarrollarse ${ }^{18}$ (Aubenque, 1981).

En síntesis el compromiso docente se asume y discute a partir de lo deseable de su presencia en ámbitos de formación y ejercicio docente, cerrando espacios para una mirada del compromiso como un imperativo. Dentro de este marco, entonces, ¿cómo es posible levantar políticas que promuevan mayores y mejores grados de implicación docente? Una respuesta iluminada por la discusión precedente no dudaría en afirmar y ratificar la imposibilidad de diseñar e implementar políticas educativas en base a un atributo o dimensión que aglutine elementos de carácter "deseable", pero no "exigible". Sin embargo, una mirada ponderada da luces y vislumbra salidas.

\section{A MODO DE CONCLUSIÓN}

Afirmar que el compromiso docente actúa como soporte de profesionalidad, sugiere un giro radical en el diseño e implementación de políticas educativas, que obliga a prestar mayor atención a una adecuada integración entre lo personal, lo profesional y lo institucional, concibiendo el quehacer profesional del profesor con un claro sentido de propósito.

De ello se desprenden al menos dos consideraciones. Por un lado, la necesidad de estructurar la formación docente, el trabajo de los formadores de formadores y el desarrollo profesional en los que el desarrollo de procesos reflexivos respecto de aspectos esenciales de la práctica pedagógica posibiliten una integración armónica entre dos dimensiones: el ser y el hacer (Korthagen, 2010). Para ello sería relevante examinar los programas de formación inicial y continua cotejando en qué medida y a través de qué estrategias se justifica e intenciona esta integración, toda vez que se reconoce la centralidad del sentido de propósito en el fortalecimiento de la capacidad de implicarse del docente.

Por otro lado, la necesidad de considerar que la construcción del sentido de propósito, aun cuando se inicia como parte de un hacer individual, tiene expresiones que incidirán tanto en lo individual como en lo colectivo y que de manera biunívoca al afectar a otros el sujeto se afectará, al transformar se transformará también, modificando tanto su identidad como lo que hemos conceptualizado como profesionalidad.

Lo ya mencionado trae como consecuencia decisiones políticas que privilegian el corto plazo, que persiguen efectos visibles, medibles y escrutables en función de agendas políticas que carecen de continuidad y que favorecen un "efectivismo" que acentúa el "hacer del profesor" en un cultivo desmedido de capacidades y competencias mesurables, que sin mayor articulación descuidan el "ser"19, lo más íntimo y esencial de aquello que el docente es y, que con demasiada frecuencia ha sido descuidado por la política educativa. Optar por

18 Miguel Ángel creía que en cada bloque de mármol en el que trabajaba existía una obra latente que él trataba de recuperar. En el caso de El David, las múltiples fracturas y fallas que tenía el bloque fueron encaminando a Miguel Ángel hacia la forma final de la escultura. Cuando se le preguntó ¿Cómo es que has hecho para salga tan perfecto? respondió "El David ya estaba dentro del mármol, lo único que hice fue quitarle lo que no le hacía falta, los excesos que no le permitían salir".

19 Analogía que Fullan (2013) utiliza para hacer referencia a la escuela y a una política que denomina Christmas tree schools toda vez que se hace alarde de innovaciones y estrategias que terminan adornando al establecimiento educacional superficialmente, pero que adolecen de profundidad y coherencia, las que pueden ser igualmente aplicadas a la política docente. 
un "efectivismo" encierra el peligro de contar con recursos adecuados para inducir mejoras en el contexto educativo, pero que si no son suministradas y administradas con la suficiente precaución, no redundan en los cambios esperados, ya que se desconecta de elementos esenciales de lo que significa ser profesor, toda vez que dicho suministro y administración es realizado por actores ajenos a la comprensión del ser del profesor.

Si bien radical, lo planteado releva la importancia que exige una mirada comprensiva y abarcadora del sistema educativo desde la subjetividad de sus actores, renovando prácticas y formas, por cierto, “desde y en" un juego armónico y equilibrado entre lo que el docente es y su actuar, reorientando los cambios en política desde los modos de actuación y relación que los profesores despliegan en espacios concretos de acción. "No basta con ensayar nuevas políticas y programas públicos u otras técnicas. Lo que está en juego es más profundo que un hecho técnico; se trata más bien, por un lado, de reorientar la propia perspectiva con la cual se mira la vida social y se orienta la conducción de los cambios" (Programa de las Naciones Unidas para el Desarrollo [PNUD], 2009: 33), y por otro, de concebir una forma distinta de desarrollo profesional docente, más allá del mero acto instrumental y mecánico de un conjunto de técnicas acordes a indicaciones de actuación externas.

Un esfuerzo de esta envergadura aboga, entre otras cosas, y tal como se argumentó previamente, a rediseñar y replantear espacios de formación y desarrollo profesional que privilegien una mirada "desde dentro", custodiando el ser del docente y del compromiso que éste establece con distintitos ámbitos de su quehacer, en función de promover una comprensión profunda y crítica de la práctica pedagógica, que estimule la emergencia de conocimientos conceptuales conectados, tanto con la dimensión personal como con la dimensión profesional de la enseñanza, a lo cual se debe agregar que "entender lo que hace efectivo el desarrollo profesional es fundamental para la comprensión, para el éxito o el fracaso de muchas reformas educativas" (Desimone, 2009: 182). Desarrollo profesional asumido como espacio que nutre tanto la construcción de la personalidad como la identidad docente, bajo el entendido que el desarrollo profesional es el proceso a través del cual los docentes, en forma individual o colegiada, revisan, renuevan y desarrollan su compromiso como agentes de cambio impulsados por propósitos morales (Day y Qing, 2010; Marcelo y Vaillant, 2009).

Desde esta perspectiva, el desarrollo profesional remite "tanto a una acción como a un efecto; refiere tanto a un proceso como a un producto" (Fuentealba, 2006: 20), lo que implica modificaciones y diversas manifestaciones a lo largo de la carrera profesional. Lo significativo es que la mayoría de profesores ante la pregunta por el quiénes son se definen a sí mismos y definen a sus pares en función del compromiso (Abd y Ngurah, 2010; Chan et al., 2008; Crosswell y Elliott, 2004; Day y Qing, 2010).

Bajo la lógica de lo expuesto, ¿sería posible pensar en atributos o condiciones de un Desarrollo Profesional que elicite el compromiso? ¿qué elementos de dicho proceso favorecen mayores grados de implicación?. Una aproximación que responde a ambas preguntas conceptualiza el desarrollo profesional desde una mirada amplia que no "atomiza" la práctica pedagógica, que no la parcela de forma arbitraria o mecanicista ${ }^{20}$. Esta entrada ofrece una comprensión de la profesión en armonía con las nociones centrales previamente trabajadas: compromiso, identidad, profesionalidad, entre otros.

20 Una visión mecanicista parte del supuesto que el docente actúa de acuerdo a las mismas leyes que las maquinas, es decir que funcionan porque funcionan, y no en base a alguna "finalidad preestablecida o algún plan interno que debe ser realizado o reflejado por su estructura (...) como ocurre con cualquier máquina, ésta se explica en términos de relaciones y no de las propiedades de los componentes" (Rosas y Sebastian, 2001: 60). 
Para la comprensión del desarrollo profesional en este marco es necesario hacer referencia a tres principios que estructuran su crecimiento: se trata de un crecimiento lento, de un crecimiento desde adentro y de un crecimiento desde una totalidad orgánica hacia otra totalidad. Este crecimiento integral se realiza normalmente siempre al mismo tiempo, pero no necesariamente en la misma proporción.

Se trata de un crecimiento lento, es decir el proceso de apropiación y adopción del repertorio propio de una profesión, y en particular en el marco de la docencia, conlleva un crecimiento paulatino que transita por estadios o etapas, los que de suyo son periódicamente interrumpidos al producirse de improviso cambios, que sí mismos son progresivos y van modificando la fisionomía del docente en construcción. Cambios que se producen en función de una multiplicidad de variables y que convocan a mayores grados de implicación al fortalecer, tensionar o mermar el compromiso docente. Así, por ejemplo, se hace visible la necesidad de contar con espacios de apoyo para aquellos que se encuentran en el período de inicio de su carrera profesional, ya que de lo contrario se limitaría la oportunidad de crecimiento del principiante.

Un crecimiento desde adentro, principio que se condice con leyes del crecimiento biológico, acentúa la expresión de desarrollo como movimiento que va desde el interior al exterior $^{21}$, en sintonía con las más diversas formas de conceptualizar el compromiso docente anteriormente expuestas, toda vez que el compromiso se define como vínculo psicológico que tiene consecuencias tanto en la actitud como en el comportamiento de las personas y que les permite voluntariamente realizar considerables esfuerzos en pos de beneficiar aquello de lo cual se es objeto de compromiso. El recorrido va desde una disposición interior a una expresión visible y externa, lo que en este caso se traduce en profesores que están dispuestos voluntariamente a entregar recursos en pos del ejercicio de la docencia (Choi y Tang, 2009). Este principio hace imposible suponer como un verdadero crecimiento lo que se obliga a partir de una acción puramente externa y que se condice con las discusiones previas respecto de la noción de estándar entre otros.

Finalmente, un crecimiento desde una totalidad orgánica hacia otra totalidad se entiende en la medida en que cada etapa de desarrollo está contenida germinalmente en su totalidad. Desde esta perspectiva, el desarrollo conoce ritmos, no se gesta en forma lineal sino que pasa por períodos en que presenta un estado de cierta armonía general, períodos que, sin embargo, son transitorios ya que el desarrollo los deja atrás para avanzar en dirección a otra etapa que, a su vez, busca también llegar a una cierta armonía en sí misma. Esto implica que no es suficiente el espacio de crecimiento individual, sino que requiere del "otro" como otra totalidad con la cual vincularse, lo que da pie para el desarrollo de espacios colectivos de construcción de sentido de propósito.

\section{REFERENCIAS}

Abd, R., \& Ngurah, G. (2010). The influence of culture on teacher commitment. Social Psychology of Education, vol.13, n.2, 185-205. doi: 10.1007/s11218-009-9109-z

Álvarez, I. y Badia, A. (2011). La identidad del profesor en contextos de educación presencia y

${ }_{21} \quad$ La ley del desarrollo próximo-distal afirma que se controlan antes las partes más próximas al eje corporal (desde adentro). Ejemplo de ello es que se controlan antes los músculos de los brazos que el movimiento de las muñecas y los dedos. Los movimientos se van haciendo crecientemente más finos, y cada vez más complejos (Palacios, Cubero, Luque, \& Mora, 1999). 
virtual. En C. Monereo y J.I. Pozo (Coords.), La Identidad en Psicología de la Educación: necesidad, utilidad y límites (pp. 213-232). Madrid: Narcea.

Aubenque, P. (1981). El problema del ser en Aristoteles. España: Taurus Ediciones.

Ávalos, B. y Sotomayor, C. (2012). Cómo ven su identidad los docentes chilenos. Perspectiva educacional, vol.51, n.1, 77-95. doi: 10.4151/07189729-Vol.51-Iss.1-Art.74

Ávalos, B., Cavada, P., Pardo, M. y Sotomayor, C. (2010). La profesión docente: temas y discusiones en la literatura internacional. Estudios Pedagógicos, vol.36, n.1, 235-263.

Atak, M. (2011). A research on the relation between organizational commitment and learning organization. African Journal of Business Management, vol.5, n.14, 5612-5616.

Bellei, C. y Valenzuela, J.P. (2011). ¿Están las condiciones para que la docencia sea una profesión de alto estatus en Chile? En S. Martinic y G. Elacqua (Eds.), ¿Fin de ciclo? cambios en la gobernanza del sistema educativo (pp. 257-285). Santiago: Unesco.

Bolívar, A. (2008). Evaluación de la práctica docente: una revisión desde España. Revista Iberoamericana de Evaluación Educativa, vol.1, n.2, 57-74.

Bolívar, A. (2010). La Lógica del Compromiso del Profesorado y la Responsabilidad del Centro Escolar. Una Revisión Actual. Revista Iberoamericana sobre Calidad, Eficacia y Cambio en Educación, vol.8, n.2, 1-25.

Cassasus, J. (2009). La Política de Reforma Basada en Estándares aplicada a Chile. Otra mala idea. En Reforma Basada en Estándares y Nuevos Ciclos Escolares: ¿Avance hacia una Educación de Calidad para Todos? (pp. 1-14). Santiago: CIAE de la Universidad de Chile. UNICEF.

Chamarro, A., Martos, V., Parrado, E. y Oberst, U. (2011). Aspectos psicológicos del baile: Una aproximación desde el enfoque de la pasión. Revista de Psicología, Ciències de l'Educació i de l'Esport, n.29, 341-350.

Chan, W., Lau, S., Nie, Y., \& Hogan, D. (2008). Organizational and personal predictors teacher commitment: the mediating role of teacher efficacy and identifications with school. American Educational Research Journal, vol.45, n.3, 597-630.

Choi, P., \& Tang, S. (2009). Teacher commitment trends: Cases of Hong Kong teachers from 1997 to 2007. Teaching and Teacher Education, vol.25, n.5, 767-777. doi: 10.1016/j.tate.2009.01.005

Consejo Económico y Social. (2011). Nuevas agendas de políticas docentes en América Latina y el Caribe: nudos críticos y criterios de acción. Buenos Aires: Naciones Unidas. ECOSOC-RMA.

Crosswell, L., \& Elliott, B. (2004, Diciembre). Committed Teachers, Passionate Teachers: the dimension of passion associated with teacher commitment and engagement. Comunicación presentada en la Australian Association for Research in Education (AARE) Annual Conference, Melbourne.

Darling-Hammond, L. (2009). Recognizing and Enhancing Teacher Effectiveness. The International Journal of Educational and Psychological Assessment, vol.3, 1-24.

Darling-Hammond, L., \& Rothman, R. (2011). Lessons Learned from Finland, Ontario, and Singapore. In Teacher and Leader Effectiveness: in High-Performing Education Systems (pp. 1-13). Washington: Alliance for Excellent Education and Stanford.

Day, C. (2006). Pasión por enseñar: la identidad personal y profesional de docente y sus valores. Madrid: Nacea.

Day, C. (2007). Committed for life? Variations in teachers' work, lives and effectiveness. Journal of Educational Change, vol.9, n.3, 243-260.

Day, C. (2009). A Passion for Quality: Teachers Who Make a Difference. Schrift voor lerarenopleiders, vol.30, n.3, 4-13.

Day, C., \& Qing, G. (2010). Teacher Emotions: Well being and effectiveness. In P. Schuktz, \& M. Zembylas (Eds.), Advances in teacher emotion research (pp. 129-153). New York: Springer.

De Azevedo, H.H. (2013). La construcción de la profesionalidad docente. Educación, vol.22, n.42, 97-115.

Desimone, L. (2009). Improving Impact Studies of Teachers' Professional Development: Toward Better Conceptualizations and Measures. Educational Researcher, vol.38, n.3, 181-199. 
Elmore, R. (2010). Mejorando la escuela desde la sala de clases. Santiago de Chile: Área de Educación Fundación Chile.

Espínola, V. y Claro, J.P. (2010). El sistema nacional de aseguramiento de la calidad: una reforma basada en estándares. En C. Bellei, D. Contreras y J.P. Valenzuela (Eds.), Ecos de la revolución pingüina. Avances, debates y silencios de la reforma educacional (pp. 51-84). Santiago: Fondo Nacional de Cultura.

Fuentealba, R. (2006). Desarrollo profesional docente: un marco comprensivo para la iniciación pedagógica de los profesores principiantes. Foro Educacional, n.10, 65-106.

Fullan, M. (2013). Change: making it happen in your school and system. ICSEI International Congress for School Effectiveness and Improvement. Santiago de Chile.

Funk, R. (2008). Búsqueda de la comprensión de la vivencia del trabajo: el trabajo como pasión (Tesis para optar al grado de Doctor en Psicología, Universidad de Chile, Escuela de Postgrado, Santiago).

Galaz, A. (2011). El profesor y su identidad profesional ¿facilitadores u obstáculos del cambio educativo? Estudios Pedagógicos, vol.37, n.2, 89-107.

Gupta, M., \& Kulshreshtha, P. (2009). Professional Commitment of the primary school teachers. The Primary Teacher, vol.34, n.3, 80-86.

Hamilton, L., Stecher, M., \& Yuan, K. (2008). Standards-Based Reform in the United States: History, Research, and Future Directions. Washington, D.C.: Center on Education Policy. RAND Corporation.

Hargreaves, A., Earl, L., Moore, S., \& Manning, S. (1998). Aprender a cambiar. Barcelona: Octaedro.

Imbarack, P. (2013). Compromiso Docente en profesores de primer ciclo básico: incidencia de la autoeficacia y resiliencia (Tesis grado académico de Doctor en Ciencias de la Educación. Facultad de Educación. Pontificia Universidad Católica de Chile).

Jacobo, H. (2003). Nuevos escenarios en la formación de los educadores mexicanos: una visión sistémica. Cuaderno de discusión, n.10, 7-41.

Klassen, R., \& Ming, C. (2010). The Occupational Commitment and Intention to Quit of Practicing and Pre-Service Teachers: Influence of Self-Efficacy, Job Stress, and Teaching Context. Comtemporary Educational Psychology, vol.36, n.2, 143-129.

Korthagen, F. (2010). La práctica, la teoría y la persona en la formación del profesorado. Revista Interuniversitaria de Formación del Profesorado, n.68, 83-102.

Kuijpers, J., Houtveen, A., \& Wubbels, T. (2010). An integrated professional development model for effective teaching. Teaching and Teacher Education, vol.26, n.8, 1687-1694.

Kwan, Y. (2012). The Relationship between the Catholic Teacher's Faith and Commitment in the Catholic High School. Catholic Education: A Journal of Inquiry and Practice, vol.15, n.2, 117-139.

Lahera, E. (2004). Introducción a las políticas públicas. Santiago de Chile: Fondo de Cultura Económica.

Larraín, J. (2005). ¿América Latina Moderna? Globalización e identidad. Santiago de Chile: LOM.

Lisbona, A., Bernabé, M., Palací, F., Gómez-Bernabeu, A., \& Martín-Aragón, M. (2012). Studying with Passion: Personal initiative and engagement. Ciencia y Trabajo, n.14, 89-95.

López de Maturana, S. (2010). Los buenos profesores: educadores comprometidos con un proyecto educativo ( $2^{\mathrm{a}}$ ed.). La serena, Chile: Universidad de la Serena.

Marcelo, C. y Vaillant, D. (2009). Desarrollo profesional docente: ¿cómo se aprende a enseñar?. Madrid: Narcea editores.

Martinic, S. (2010). Cambios en las Regulaciones del Sistema Educativo. ¿Hacia un Estado Evaluador? En S. Martinic y G. Elacqua (Eds.), ¿Fin de ciclo? cambios en la gobernanza del sistema educativo (pp. 55-78). Santiago: UNESCO.

Ministerio de Educación de Chile. (2011). Estándares orientadores para egresados de carreras de pedagogía en Educación Básica. Santiago de Chile: Autor. 
Moè, A., Pazzaglia, F., \& Ronconi, L. (2010). When being able is not enough. The combined value of positive affect and self-efficacy for job satisfaction in teaching. Teaching and Teacher Education, vol.26, n.5, 1145-1153.

Monereo, C. y Badia, A. (2011). Los heterónimos del docente: Identidad, selfs y enseñanza. En C. Monereo y J.I. Pozo (Coords.), La Identidad en Psicología de la Educación: necesidad, utilidad y límites (pp. 57-76). Madrid: Narcea.

Nias, J. (1981). Commitment and motivation in primary school teachers. Educational Review, vol.33, n.3, 181-190.

Palacios, J., Cubero, R., Luque, A. y Mora, J. (1999). Desarrollo psicológico y educación. Madrid: Alianza.

Palmer, J. (2007). The courage to teach: exploring the inner landscape of a teacher's life (10 ed.). San Francisco: Jossey Bass.

Pérez-Gómez, A., Barquín, J. y Angulo, J. (Eds.). (1999). Desarrollo profesional docente: política, investigación y práctica. Madrid: Akal.

PNUD. (2009). Desarrollo humano en Chile: la manera de hacer las cosas. Santiago de Chile: Autor.

Razak, N.A., Darmawan, I., \& Keeves, J. (2009). Teacher commitment. In L. Saha, \& A. Dworkin, (Eds.), International Handbook of Research on Teachers and Teaching (pp. 343-360). Springer US. doi: 10.1007/978-0-387-73317-3

Rosas, R., y Sebastian, C. (2001). Piaget, Vigotsky y Maturana: constructivismo a tres voces. Santiago: AIQUE.

Sammons, P., Day, C., Kington, A., Gu, Q., Stobart, G., \& Smees, R. (2007). Exploring variations in teachers' work, lives and their effects on pupils: key findings and implications from a longitudinal mixed-method study. British Educational Research Journal, vol.33, n.5, 681-701.

Santoro, N., Pietsch, M., \& Borg, T. (2012). The passion of teaching: learning from an older generation of teachers. Journal of Education for Teaching: International research and pedagogy, vol.38, n.5, 585-595.

Selamat, N., \& Nordin, N. (2014). Teacher's commitment and emotional intelligence: evidence from Malaysian schools. International journal of Science Commerce and Humanities, vols.2-3, 49-59.

Sisto, V. (2011). Nuevo profesionalismo y profesores: una reflexión a partir del análisis de las actuales políticas de 'profesionalización' para la educación en Chile. Signo y Pensamiento, vol.31, n.59, 178-192.

Tang, S., \& Choi, P. (2011). Satisfied and Dissatisfied Commitment: Teachers in Three Generations. Australian Journal of Teacher Education, vol.36, n.7, 44-75.

Tigchelaar, A., Brouwer, N., \& Korthagen, F. (2008). Crossing horizons: Continuity and change during second-career teachers' entry into teaching. Teaching and Teacher Education, vol.24, n.6, 1530-1550.

UNESCO. (2011). Nueva agenda de políticas docentes en América latina y el Caribe: nudos críticos y criterios de acción. Santiago de Chile: Oficina Regional de Educación para América Latina y el Caribe (OREALC/UNESCO Santiago).

Vallerand, R. (2012). The role of passion in sustainable psychological well-being. Research and Practice, vol.2, n.1. doi:10.1186/2211-1522-2-1

Vallerand, R., Paquet, Y., Philippe, F., \& Charest, J. (2010). On the Role of Passion for Work in Burnout: A Process Model. Journal of Personality, vol.78, n.1, 289-312. doi: 10.1111/j.14676494.2009.00616.x.

Van de Berg, R. (2002). Teachers' Meanings regarding Educational Practice. Review Of Educational Research, vol.72, n.4, 577-625.

Vezub, L. (2007). La formación y el desarrollo profesional docente frente a los nuevos desafíos de la escolaridad. Profesorado Revista de curriculum y formación de profesorado, vol.11, n.1, 1-23. Recuperado desde http://www.redalyc.org/articulo.oa?id=56711102 
